\title{
Prevalence and antifungal susceptibility of Candida albicans causing vaginal discharge among pregnant women in Lebanon
}

Nahed Ghaddar ${ }^{1}$, Elie Anastasiadis ${ }^{2,3}$, Rawad Halimeh ${ }^{2}$, Ali Ghaddar ${ }^{4}$, Rita Dhar ${ }^{7}$, Wadha AlFouzan ${ }^{5,7}$, Hoda Yusef ${ }^{1}$ and Mira El Chaar ${ }^{6 *}$

\begin{abstract}
Background: Vaginal candidiasis is frequent in pregnant women and is associated with sepsis and adverse neonatal outcomes. This study determined the prevalence of candida species in symptomatic pregnant women and evaluated the antifungal susceptibility profile of the isolated Candida strains. It also aimed to explore whether Candida species predicts gestational complications and adverse neonatal outcomes.

Methods: A total of 258 pregnant women with vaginal discharge at 35 to 37 week of gestation participated in this study. Vaginal swabs from these patients were collected at various obstetrics and gynecology clinics in Lebanon for a period of 14 months. Candida isolates were identified at species level and antifungal susceptibility of Candida albicans to fluconazole (FCZ), amphotericin B (AMB), itraconazole (ICZ) and voriconazole (VCZ) was determined by the agar-based E-test method.

Results: Among 258 women tested, 100 (39\%) were positive for Candida species. C. albicans, C. glabrata and C. krusei were isolated from 42,41 and 17\% of the women, respectively. C. albicans was significantly associated only with gestational diabetes while C. krusei or C. glabrata had significant positive associations with other gestational complications. The antifungal susceptibility tests of $C$. albicans isolates revealed 97.5, 90, 87.5 and 97.5\% susceptibility to AMB, FCZ, ICZ and VCZ, respectively.

Conclusion: The current study revealed high incidence of both C. albicans and non-C. albicans Candida strains causing vulvovaginitis among pregnant women in Beirut, Lebanon. Candida screening as antenatal follow up is advised to minimize the risk of adverse neonatal outcome or gestational complications.
\end{abstract}

Keywords: Candida albicans, Vulvovaginitis, Prevalence, Pregnant women, Antifungal susceptibility

\section{Background}

Candida species, which are part of the normal flora in the vulvovagina, may cause opportunistic infections under various circumstances that compromise host immunity. Candida spp. subsist in symbiotic relationship with vaginal microbiota, therefore asymptomatic colonization is common and may persist for years. The rate of genital Candida colonization ranges from $20 \%$ in asymptomatic young women to up to $30 \%$ in pregnant women [1-6].

\footnotetext{
* Correspondence: mira.elchaar@balamand.edu.lb

${ }^{6}$ Department of Microbiology, Health Sciences Center, Kuwait University, Jabriya, Kuwait

Full list of author information is available at the end of the article
}

The risk factors associated with increased rate of vulvovaginal candidiasis (VVC) in pregnant women are immunologic alterations, increased estrogen levels, and increased vaginal glycogen production mechanism [3].

VVC is the result of Candida albicans in $85-95 \%$ of cases whereas incidence rate of Non-C. albicans Candida $(\mathrm{NCAC})$ in pregnant women and non-pregnant women is less than $10 \%$ as described in previous studies [7].

Treatment of VVC is recommended only in the presence of symptoms since over $20 \%$ of women may have yeast as part of their natural vaginal microbiome and are asymptomatic [8]. However, pregnant women may have severe and prolonged symptoms of VVC requiring longer courses of therapy [9]. Recent studies have shown an

(c) The Author(s). 2020 Open Access This article is distributed under the terms of the Creative Commons Attribution 4.0 International License (http://creativecommons.org/licenses/by/4.0/), which permits unrestricted use, distribution, and reproduction in any medium, provided you give appropriate credit to the original author(s) and the source, provide a link to the Creative Commons license, and indicate if changes were made. The Creative Commons Public Domain Dedication waiver (http://creativecommons.org/publicdomain/zero/1.0/) applies to the data made available in this article, unless otherwise stated. 
increase in the development of drug-resistance among C. albicans, less is known about the burden and effects of drug resistant fungal infections.

Candidiasis in newborns has been associated with increased risk of pregnancy complications, such as premature rupture of membranes, preterm labor, chorioamnionitis, and congenital cutaneous candidiasis. Colonization with Candida spp. in neonates may occur by vertical transmission from the mother during the perinatal period or by horizontal transmission in the nursery or the neonatal intensive care unit (NICU) [10-12]. It has been shown that 5 to $30 \%$ of all colonized preterm neonates will develop invasive Candida infection (ICI) during their stay in the NICU [13-15]. C. albicans was shown to play a major role in neonatal colonization in the first days of life and were also documented in a group of premature infants [16].

The epidemiology of antifungal resistance among $C$. albicans in pregnant women in Lebanon remains poorly reported. Therefore, the objective of this study was to determine the prevalence of Candida species in symptomatic pregnant women with vaginal discharge at 35 to 37 weeks of gestation and to evaluate the antifungal susceptibility profile of the isolated strains of $C$. albicans. In addition, the study evaluated the association between the presence of Candida species and gestational complications and outcomes.

\section{Methods}

\section{Study sample and procedure of collection}

In this study, a cross-sectional design was adopted for determining the prevalence of Candida species in Lebanese pregnant women. Clinical samples were collected from 258 pregnant women with vaginal discharge in three obstetrics and gynecology clinics in Lebanon during a period of 14 months (June 2015-July 2016). Women were approached by a registered gynecologist who explained the objectives of the study and asked them to participate. Participation was voluntary and anonymous. Two vaginal swabs were collected from each patient. The samples were stored in Stuart media (Oxoid, UK) at room temperature and transported to the clinical diagnostic laboratory.

\section{Data collection}

Socio-demographic data, clinical status and gestational history of $165(64 \%)$ patients were collected through a questionnaire that included information about mothers' risk factors for adverse neonatal outcomes such as gestational diabetes, previous miscarriage, anemia and recurrent urinary tract infections (UTI). The 165 participants were followed up after delivery to gather information about delivery time, delivery type, induced labor, gestational complications (intrahepatic cholestasis, mitral valve prolapse, asthma, hypothyroidism, oligohydramnios and gestational thrombocytopenia) and neonatal outcomes (newborn height, weight and apgar score).

\section{Literature search}

To compare the distribution of Candida species isolated from women genital tract in different countries, a Pubmed search was performed that included articles published in the last 10 years [17-23]. Fifteen articles were selected and summarized in Fig. 1.

\section{Culture and identification}

Vaginal swabs were cultured on both Sabouraud dextrose agar (SDA) and Chromatic Candida medium (Liofilchem, Italy). The latter allows the selective isolation and differentiation of Candida spp. based on colony color and morphology; it has been well documented in previous studies as for its high sensitivity and specificity for the identification of the most commonly encountered Candida spp. [24-26]. Both plates were incubated at $37^{\circ} \mathrm{C}$ for 48 to $72 \mathrm{~h}$. The chromatic characteristics of the colonies were the following: green colonies were identified as $C$. albicans, creamy colored colonies as C. glabrata, and pink with a whitish border colonies as $C$. krusei. All isolates were confirmed by API 20 C AUX strip (BioMerieux, Marcy l'Etoile, France). Further phenotypic testing was done to differentiate between $C$. dubliniensis and $C$. albicans by growing the germ tube positive yeast isolates at $45^{\circ} \mathrm{C}$ on SDA for up to 10 days. C. albicans isolates were identified by their ability to grow at $45^{\circ} \mathrm{C}$.

\section{Antifungal susceptibility testing}

The in vitro activity of the antifungal agents against each isolate was determined by the E-test (HiMedia, Mumbai, India) in accordance with the manufacturer's instructions. The E-test strips of fluconazole (FCZ; 0.016 $256 \mu \mathrm{g} / \mathrm{mL}$ ), itraconazole (ICZ; $0.002 \sim 32 \mu \mathrm{g} / \mathrm{mL}$ ), voriconazole (VCZ; $0.002 \sim 32 \mu \mathrm{g} / \mathrm{mL}$ ), and amphotericin B (AMB; $0.002 \sim 32 \mu \mathrm{g} / \mathrm{mL}$ ) were used [27]. Interpretive susceptibility criteria for antifungal breakpoints were adapted from the Clinical and Laboratory Standards Institute (CLSI), 2017 (21). The breakpoints used for $C$. albicans are: $\mathrm{FCZ}(\mathrm{S} \leq 2 ; \mathrm{SDD}=4 ; \mathrm{R} \geq 8) ; \mathrm{VCZ}(\mathrm{S} \leq 0.12$; $\mathrm{R} \geq 1)$, ICZ $(\mathrm{S} \leq 0.12 ; \mathrm{R} \geq 1)$ and $\mathrm{AMB}(\mathrm{S} \leq 2 ; R>2)$. For quality control, C. albicans (ATCC 10231) was used as reference strain and tested simultaneously with the clinical isolates.

\section{Statistical analysis}

The presence of Candida species was correlated with the newborn height, weight and Apgar score (overall assessment of new born well-being used immediately following the delivery of the baby) as dependent variables using linear multiple regression analysis. The models took into consideration to control other possible confounding 


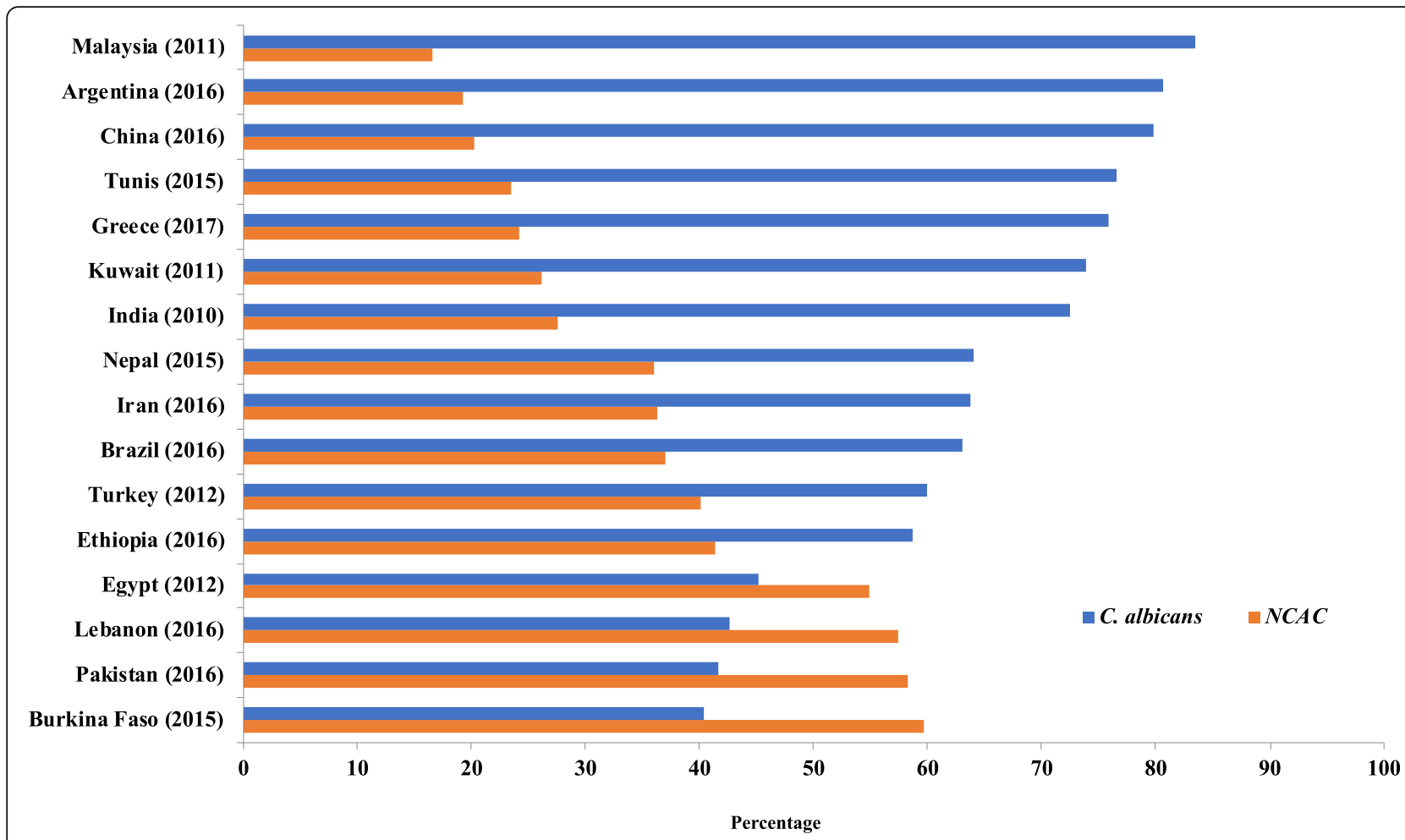

Fig. 1 Distribution of Candida species isolated from vaginal swabs of women from various countries after 2010

effect of various independent variables including mother's age, mother's education, delivery type, delivery week, induced labor, recurrent urinary tract infection (UTI), gestational diabetes, anemia and other gestational complications. Statistical significance was calculated using $p$-value and confidence intervals. The presence of Candida species effect on categorical outcome variables (Gestational diabetes, vaginal discharge, induced labor and recurrent UTI) was explored by comparing frequencies using the test of independence Chi-square. $P$ values were computed considering $p \leq 0.05$ as significant results.

\section{Results}

The socio-demographic characteristics of 165 respondents are summarized in Table 1; $49 \%$ of women who participated were between the ages of 36 to 40 years old. The majority completed their university degree (62.4\%). The rate of normal vaginal delivery was 43.6 and $69.7 \%$ of women had labor induction. Gestational complications occurred in $59.1 \%$ of women and $25.5 \%$ of women had gestational diabetes mellitus. Anemia was reported in $14.5 \%$ of women.

The data presented in the table are number (\%) of patients. Missing are missing data since patients' information were not provided; those were excluded from the analysis. Gestational complications include intrahepatic cholestasis, mitral valve prolapse, asthma, hypothyroidism, oligohydramnios and gestational thrombocytopenia.
Table 1 Socio-demographic characteristics of 165 respondents

\begin{tabular}{lll}
\hline Age group & 20-25 years & $19(11.5 \%)$ \\
& 26-30 years & $14(8.5 \%)$ \\
& $36-40$ years & $49(29.7 \%)$ \\
& $\geq 41$ years & $21(49.1 \%)$ \\
Education status & Primary & $32.2 \%)$ \\
& Secondary & $30(19.4 \%)$ \\
Delivery type & University & $103(62.4 \%)$ \\
& Normal & $72(43.6 \%)$ \\
Cesarean section & $24(14.5 \%)$ \\
Induced labor & Missing & $69(41.8 \%)$ \\
Recurrent UTI & Yes & $50(30.3 \%)$ \\
Gestational complications & No & $115(69.7 \%)$ \\
& Yes & $4(2.4 \%)$ \\
Anemia & No & $161(97.6 \%)$ \\
& Yes & $97(59.1 \%)$ \\
& No & $67(40.9 \%)$ \\
& Missing & $1(0.6 \%)$ \\
Yes & $42(25.5 \%)$ \\
No & $123(74.5 \%)$ \\
& Yes & $141(85.5 \%)$ \\
\hline
\end{tabular}


Among the cultures from the 258 women tested, 100 (39\%) were positive for Candida species.

C. albicans was isolated from $42 \%$ of the women $(N=$ 42 ) and NCAC from the remaining 58\%. Figure 1 summarizes the distribution of Candida species isolated from vaginal swabs from population-based studies conducted in different countries including our study over the last decade. China, Brazil, Tunis, Kuwait, India, Greece and Turkey have reported that $C$. albicans remains the most commonly isolated yeast $(60-80 \%)$ in women diagnosed with VVC [17-23]. On the other hand, an increasing trend in the occurrence of NCAC (58-60\%) over time has also been observed in Pakistan and Burkina Faso $[28,29]$. The main identified NCAC in our study were C. glabrata $(71 \%, N=41)$ followed by C. krusei $(29 \%$, $N=17)$. Four women were co-infected with both $C$. albicans and C. glabrata. All of the three identified Candida species were isolated from women in the age group 3040 years.

The observed susceptibility rates of $C$. albicans isolates to AMB, FCZ, ICZ and VCZ were 97.5, 90, 87.5 and $97.5 \%$, respectively. $\mathrm{MIC}_{50}$ and $\mathrm{MIC}_{90}$ of the antifungal agents tested against 40 strains of C. albicans are presented in Table 2. Two of the isolates were lost during processing, Although ICZ presented the lowest $\mathrm{MIC}_{90}$ value of $0.125 \mu \mathrm{g} / \mathrm{mL}$, it showed highest resistance rate (12.5\%) among all the agents tested.

$\mathrm{MIC}_{50}=$ Minimum Inhibitory Concentration required to inhibit the growth of $50 \%$ of organisms. $\mathrm{MIC}_{90}=$ Minimum Inhibitory Concentration required to inhibit the growth of $90 \%$ of organisms. MIC range is the range of the lowest and highest MIC values obtained from 40 C. albicans isolates tested. Percentage resistance is the percentage of isolates resistant to a specific antifungal drug.

The association between the presence of Candida species, isolated from 48 women who filled the questionnaire, was assessed with preterm delivery, delivery type, gestational complications, gestational diabetes, recurrent UTI infection and induced labor (Table 3). C. albicans was significantly associated only with patients with gestational diabetes; $33 \%$ of $C$. albicans positive and $24 \%$ of NCAC participants had gestational diabetes $(p=0.04)$. C. albicans had non-significant associations with gestational complications, induced labor and recurrent UTI. On the other hand, the presence of C. krusei and C. glabrata had strong

Table 2 Ranges of MICs, MIC50 and MIC90 and percentage resistance in $40 \mathrm{C}$. albicans isolates

\begin{tabular}{lllll}
\hline Antifungal drugs & MIC 50 & MIC 90 & MIC Range & Percentage resistance \\
\hline Amphotericin B & 0.5 & 1 & $0.38-3.00$ & $2.5 \%$ \\
Fluconazole & 2 & 6 & $0.047-32$ & $10.0 \%$ \\
Itraconazole & 0.125 & 0.125 & $0.032-32$ & $12.5 \%$ \\
Voriconazole & 0.094 & 1 & $0.032-256$ & $2.5 \%$ \\
\hline
\end{tabular}

significant association with premature delivery and gestational complications $(p<0.05)$ : $94 \%$ of women with $C$. glabrata and $71.4 \%$ of women with C. krusei had gestational complications compared to 28.4 and $29.7 \%$ of women who did not have C. glabrata and C. krusei respectively ( $p$-value $\leq 0.001$ ). No significant associations were observed between the isolated Candida species and induced labor or recurrent UTI (Table 3).

Results of the three multiple regression models with neonatal outcomes (weight, height and Apgar score) as dependent variables are displayed in Table 4. Results revealed significant positive association between delivery time and neonatal height and significant negative association between $\mathrm{C}$-section and height. Height increased $0.41 \mathrm{~cm}$ with 1 week increase in delivery time $(p=0.001)$ and decreased $0.46 \mathrm{~cm}$ with $\mathrm{C}$-section $(p=0.002)$. Height also decreased with the presence of all identified Candida species. This reduction was statistically significant in both C. krusei or C. glabrata infections (Beta $=-0.46$, $p=0.05$ for $C$. albicans and Beta $=-0.77 ; p=0.006$ ). The other covariates did not yield significant associations with height. Neonatal weight had significant positive association with delivery time and significant negative association with $\mathrm{C}$-section. There was $0.32 \mathrm{~g}$ increase in weight with an additional delivery week $(p=0.01)$ and $0.34 \mathrm{~g}$ decrease in weight with C-section $(p=0.02)$. Although weight decreased with the presence of Candida species (C. albicans: Beta $=0.16, C$. krusei or $C$. glabrata: Beta $=0.43$ ), this reduction was not statistically significant. The other covariates did not yield significant associations with weight. Apgar score did not show significant correlation with the presence of any Candida species or with any of the other independent variables.

\section{Discussion}

The prevalence of Candida species causing vaginitis is pregnant women vary from one population to another. In our study, 39\% of participating women were infected by Candida species. NCAC were more frequently isolated (58\%) than C. albicans (42\%). NCAC were also shown to increase in non vaginal clinical samples isolated from Lebanon; that was observed in a previous retrospective study published where the authors have shown that among all Candida strains isolated, C. albicans rates had decreased from $86 \%$ in 2005 to around $60 \%$ in 2014 . However, the NCAC rates increased from $14 \%$ in 2005 to around $40 \%$ in 2014 , comprising mainly of C. tropicalis, C. glabrata, and C. parapsilosis [30]. Recent emergence of NCAC, such as $C$. glabrata and $C$. krusei has been seen in the post FCZ era and in settings with azole selection pressure [31]. Worldwide, there is a variation in the distribution of Candida spp. identified from vaginal swabs and depends largely on the location as well as the population studied (Fig. 1). 


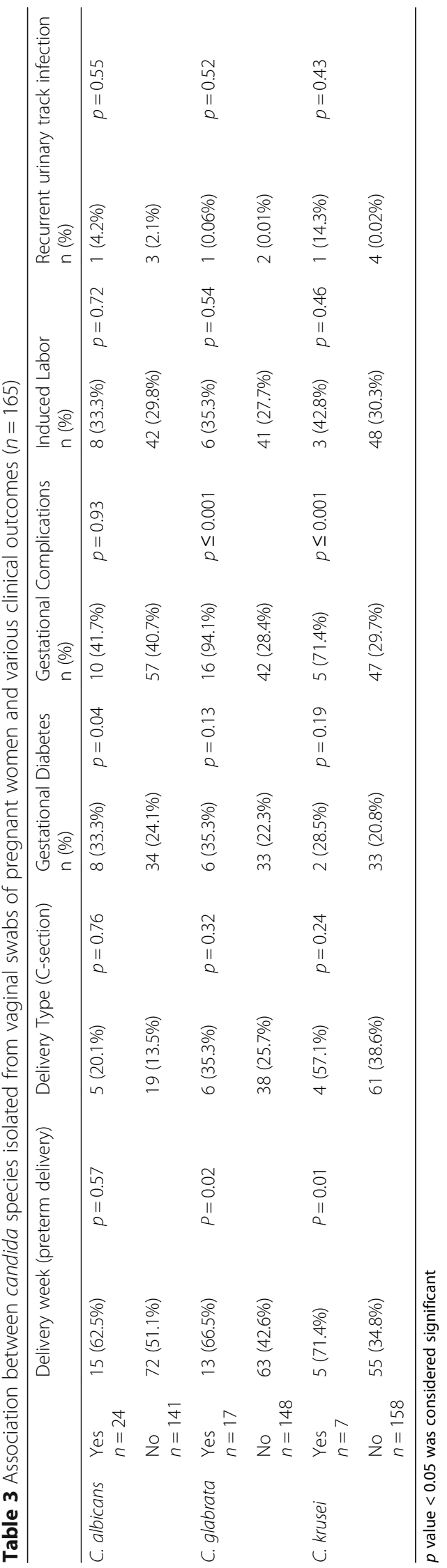




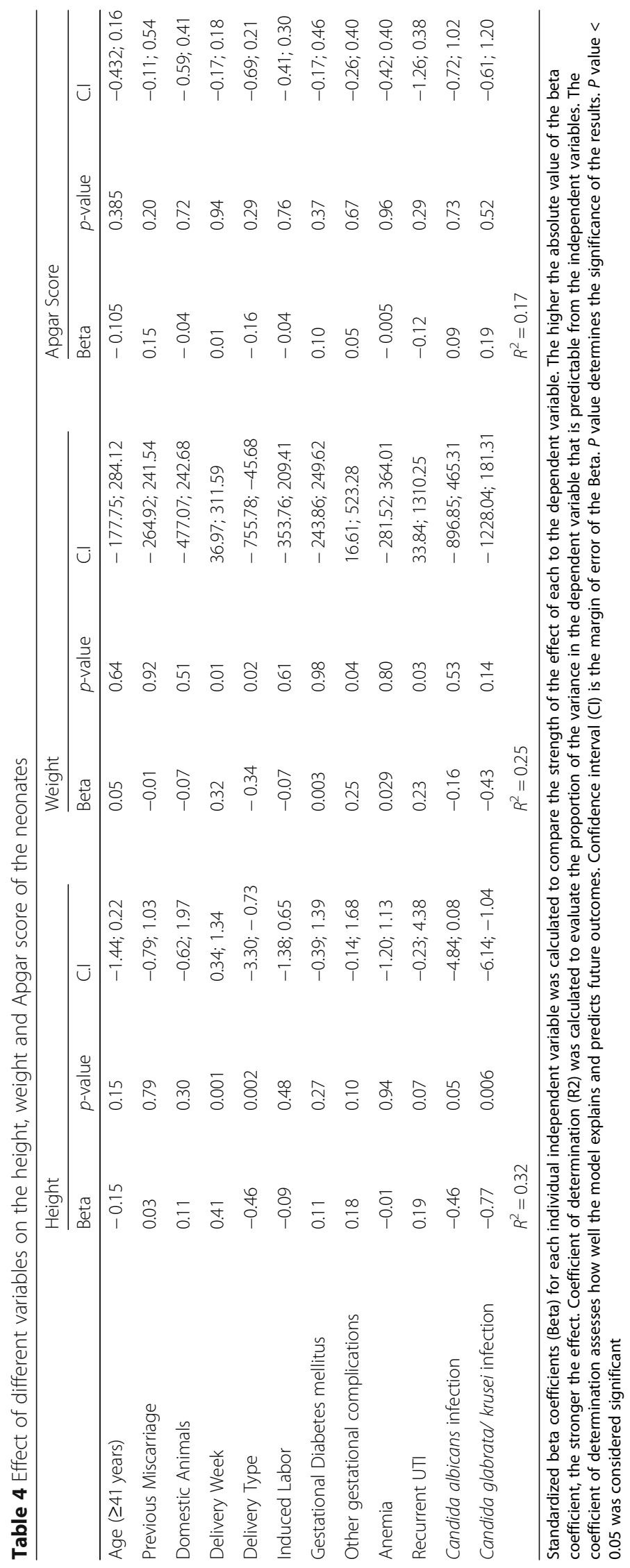


Treatment of vaginal candidiasis is successfully achieved by use of azoles [32]. NCAC related disease is less likely to respond to azole therapy, alternative treatment with $\mathrm{AMB}$ suppositories with or without topical azole is recommended. In the current study, isolates showed high susceptibility to AMB (97.5\%) and this observation has been corroborated by studies done in various other countries including Lebanon [30, 33-35]. Resistance rates of C. albicans to VCZ, FCZ, and ICZ and in this study were $2.5,10$, and $12.5 \%$, respectively, which are in contrast to earlier data from Lebanon reporting 3 to $6 \%, 0$ to 6 , and $38 \%$ resistance, respectively [30]. However, despite high susceptibility rates against FCZ and VCZ in the previous study, their $\mathrm{MIC}_{90}$ showed an elevated trend over 10 year of study period [30]. The increase in azole resistance in our study can be attributed to the frequent empiric prescription of FCZ for sporadic VVC, which may result in FCZ-resistant $C$. albicans causing recurrent VVC infection to emerge [36]. Identification of the most common molecular mechanism of resistance among our clinical isolates would help in understanding if there is any spread of resistance gene between C. albicans and NCAC. Since through vertical or horizontal transmission, $5-30 \%$ of all colonized preterm neonates may develop invasive Candida infection [13-15], prophylaxis with antifungal agents in this group of patients has proven effective in preventing such an infection. However, an increase in MIC against antifungal agents may have major consequences resulting in poor outcomes and higher mortality rate among neonates with invasive candida infection.

Although treatment of asymptomatic pregnant women with Candida colonization in the genital tract is not yet recommended, some countries such as Germany have started to implement the process of screening and treatment of women found to be colonized vaginally by Candida spp. or those who present with VVC in the third trimester [37]. In Lebanon, unlike group B streptococcus (GBS), routine screening for the presence of Candida spp. in pregnant women in the third week of gestation is not considered as part of a routine surveillance by the obstetricians. Since invasive candidiasis in neonates is becoming a serious and common cause of late onset sepsis, with mortality rates reaching as high as $25-35 \%$ [10], screening simultaneously for both GBS and Candida spp. in pregnant women would reduce the rate of sepsis, meningitis, oral thrush and diaper dermatitis in newborns with these organisms acquired during vaginal delivery.

It is reported that vaginitis in pregnancy is related to adverse perinatal outcome [38]. In the current study, we aimed to find correlation between the presence of candidiasis and pregnancy outcome. Our results showed that height decreased with the presence of Candida species. This reduction was statistically significant in the presence of C. krusei or C. glabrata. However no effect was observed on the weight of the baby. This finding was consistent with a study done previously in Iran where they found no association between vaginal Candida colonization and low birth weight [39]. The current study has also shown that Candida species cause gestational complications which is also in agreement with a previous study done in China [40].

Among the different studied variables which may be affected by Candida, such as gestational complications, gestational diabetes, vaginal discharge, induced labor and recurrent UTI, the present study confirmed that the presence of $C$. albicans was significantly associated with women with gestational diabetes and both $C$. krusei and C. glabrata on women with gestational complications. Future case control studies should be performed to compare the clinical outcome of pregnant women infected with any microorganism versus non infected women.

The study has potential limitations which include the lack of screening of other pathogens in pregnant women; these may have an impact on pregnancy outcomes. Case control studies should be also implemented to determine if exposure to Candida species has an association with pregnancy outcomes.

\section{Conclusions}

In conclusion, increasing rates of NCAC strains among pregnant women in Lebanon should be looked at as both novel and alarming. Extensive surveillance studies should be done on all clinical specimens yielding significant growth of Candida spp. and the effect of resistance pattern on ICI. As a consequence of selective pressure, emergence of drug resistance is inevitable. Therefore future studies should focus on the emergence of drug-resistant Candida strains and their frequencies. The susceptibility pattern of $C$. albicans to antifungal agents varies with region and would require constant monitoring of any unusual increase in resistance.

\section{Abbreviations \\ AMB: Amphotericin B; Beta: Standardized beta coefficients; Cl: Confidence interval; CLSI: Clinical and Laboratory Standards Institute; FCZ: Fluconazole; GBS: Group B streptococcus; ICZ: Itraconazole; NCAC: Non-C. albicans Candida; NICU: Neonatal intensive care unit; R2: Coefficient of determination; SDA: Sabouraud dextrose agar; UTI: Urinary tract infections; VCZ: Voriconazole; WC: Vulvovaginal candidiasis}

\section{Acknowledgements}

None

\section{Authors' contributions}

NG was responsible for the study design, performed and analyzed the experiments in addition to data analysis and writing up the manuscript. MEC was responsible for the study design, supervised and analyzed the experiments and was responsible for writing up the manuscript. AE and $\mathrm{RH}$ were responsible for sampling and clinical interpretation. AG was responsible for the epidemiological and statistical analysis of the data. RD, WAF and $\mathrm{HY}$ revised the manuscript. All authors reviewed and approved the manuscript. 


\section{Funding}

No funding

\section{Availability of data and materials}

The datasets used and/or analysed during the current study are available from the corresponding author on reasonable request.

\section{Ethics approval and consent to participate}

This study was approved by the institutional review board of Beirut Arab University under IRB\# 0041-S-P-0336. A written informed consent was obtained from all eligible women before entering the study.

\section{Consent for publication}

Not applicable

\section{Competing interests}

The authors declare that they have no competing interests.

\section{Author details}

'Faculty of Science, Biological Sciences Department, Beirut Arab University, Beirut, Lebanon. ${ }^{2}$ Department of Obstetrics and Gynecology, Saint George Hospital, Beirut, Lebanon. ${ }^{3}$ Faculty of Medicine, University of Balamand, Beirut, Lebanon. ${ }^{4}$ Department of Biomedical Sciences, Lebanese International University, Beirut, Lebanon. ${ }^{5}$ Microbiology Unit, Department of Laboratories, Farwania Hospital, Sabah Al Nasser, Kuwait. ' Department of Microbiology, Health Sciences Center, Kuwait University, Jabriya, Kuwait. ${ }^{7}$ Faculty of Health Sciences, University of Balamand, P.O.Box 166378 Ashrafieh, Beirut 1100-2807, Lebanon.

Received: 18 July 2019 Accepted: 24 December 2019

\section{Published online: 13 January 2020}

\section{References}

1. Aguin TJ, Sobel JD. Vulvovaginal candidiasis in pregnancy. Curr Infect Dis Rep. 2015;17(6):462.

2. Beigi RH, Meyn LA, Moore DM, Krohn MA, Hillier SL. Vaginal yeast colonization in nonpregnant women: a longitudinal study. Obstet Gynecol. 2004;104(5 Pt 1):926-30.

3. Goncalves B, Ferreira C, Alves CT, Henriques M, Azeredo J, Silva S. Vulvovaginal candidiasis: epidemiology, microbiology and risk factors. Crit Rev Microbiol. 2016;42(6):905-27.

4. Holland J, Young ML, Lee O. S CAC: Vulvovaginal carriage of yeasts other than Candida albicans. Sex Transm Infect. 2003;79(3):249-50.

5. Larsen B. Vaginal flora in health and disease. Clin Obstet Gynecol. 1993: 36(1):107-21.

6. van Schalkwyk J, Yudin MH. Vulvovaginitis: screening for and management of trichomoniasis, vulvovaginal candidiasis, and bacterial vaginosis. J Obstet Gynaecol Can. 2015:37(3):266-74

7. Sobel JD. Vulvovaginal candidosis. Lancet (London, England). 2007; 369(9577):1961-71.

8. Farr A, Kiss H, Holzer I, Husslein P, Hagmann M, Petricevic L. Effect of asymptomatic vaginal colonization with Candida albicans on pregnancy outcome. Acta Obstet Gynecol Scand. 2015;94(9):989-96.

9. Cotch MF, Hillier SL, Gibbs RS, Eschenbach DA. Epidemiology and outcomes associated with moderate to heavy Candida colonization during pregnancy. Vaginal infections and prematurity study group. Am J Obstet Gynecol. 1998; 178(2):374-80

10. Wadile RG, Bhate VM. Study of clinical spectrum and risk factors of neonatal candidemia. Indian J Pathol Microbiol. 2015:58(4):472-4

11. Filippidi A, Galanakis E, Maraki S, Galani I, Drogari-Apiranthitou M, Kalmanti M, Mantadakis E, Samonis G. The effect of maternal flora on Candida colonisation in the neonate. Mycoses. 2014;57(1):43-8.

12. Leibovitz E. Strategies for the prevention of neonatal candidiasis. Pediatr Neonatol. 2012:53(2):83-9.

13. Manzoni P, Mostert M, Jacqz-Aigrain E, Stronati M, Farina D. Candida colonization in the nursery. J Pediatr. 2012;88(3):187-90.

14. Benjamin DK Jr, Stoll BJ, Fanaroff AA, McDonald SA, Oh W, Higgins RD, Duara S, Poole K, Laptook A, Goldberg R. Neonatal candidiasis among extremely low birth weight infants: risk factors, mortality rates, and neurodevelopmental outcomes at 18 to 22 months. Pediatrics. 2006; 117(1):84-92
15. Steinbach WJ, Roilides E, Berman D, Hoffman JA, Groll AH, Bin-Hussain I, Palazzi DL, Castagnola E, Halasa N, Velegraki A, et al. Results from a prospective, international, epidemiologic study of invasive candidiasis in children and neonates. Pediatr Infect Dis J. 2012;31(12):1252-7.

16. Waggoner-Fountain LA, Walker MW, Hollis RJ, Pfaller MA, Ferguson JE. 2nd, Wenzel RP, Donowitz LG: vertical and horizontal transmission of unique Candida species to premature newborns. Clin Infect Dis. 1996;22(5):803-8.

17. Zhai Y, Liu J, Zhou L, Ji T, Meng L, Gao Y, Liu R, Wang X, Li L, Lu B, et al. Detection of Candida species in pregnant Chinese women with a molecular beacon method. J Med Microbiol. 2018;67:783-9.

18. Mtibaa L, Fakhfakh N, Kallel A, Belhadj S, Belhaj Salah N, Bada N, Kallel K. Vulvovaginal candidiasis: etiology, symptomatology and risk factors. J Mycol Med. 2017:27(2):153-8

19. Alfouzan W, Dhar R, Ashkanani H, Gupta M, Rachel C, Khan ZU. Species spectrum and antifungal susceptibility profile of vaginal isolates of Candida in Kuwait. J Mycol Med. 2015;25(1):23-8

20. Dharmik PG, Gomashe AV, Upadhyay VG. Susceptibility pattern of various azoles against Candida species causing vulvovaginal candidiasis. J Obstet Gynaecol India. 2013;63(2):135-7.

21. Kalkanci A, Guzel AB, Khalil II, Aydin M, Ilkit M, Kustimur S. Yeast vaginitis during pregnancy: susceptibility testing of 13 antifungal drugs and boric acid and the detection of four virulence factors. Med Mycol. 2012;50(6):585-93.

22. Brandolt TM, Klafke GB, Goncalves CV, Bitencourt LR, Martinez AM, Mendes JF, Meireles MC, Xavier MO. Prevalence of Candida spp. in cervical-vaginal samples and the in vitro susceptibility of isolates. Braz J Microbiol. 2017; 48(1):145-50.

23. Maraki S, Mavromanolaki VE, Stafylaki D, Nioti E, Hamilos G, Kasimati A Epidemiology and antifungal susceptibility patterns of Candida isolates from Greek women with vulvovaginal candidiasis. Mycoses. 2019;62(8):692-7.

24. Madhavan P, Jamal F, Chong PP, Ng KP. Identification of local clinical Candida isolates using CHROMagar Candida as a primary identification method for various Candida species. Trop Biomed. 2011:28(2):269-74.

25. Daef E, Moharram A, Eldin SS, Elsherbiny N, Mohammed M. Evaluation of chromogenic media and seminested PCR in the identification of Candida species. Braz J Microbiol. 2014;45(1):255-62.

26. Ouanes A, Kouais A, Marouen S, Sahnoun M, Jemli B, Gargouri S. Contribution of the chromogenic medium CHROMagar((R))Candida in mycological diagnosis of yeasts. J Mycol Med. 2013;23(4):237-41.

27. Song YB, Suh MK, Ha GY, Kim H. Antifungal susceptibility testing with Etest for Candida species isolated from patients with Oral candidiasis. Ann Dermatol. 2015;27(6):715-20.

28. Khan M, Ahmed J, Gul A, Ikram A, Lalani FK. Antifungal susceptibility testing of vulvovaginal Candida species among women attending antenatal clinic in tertiary care hospitals of Peshawar. Infect Drug Resist. 2018:11:447-56.

29. Sangare I, Sirima C, Bamba S, Zida A, Cisse M, Bazie WW, Sanou S, Dao B, Menan H, Guiguemde RT. Prevalence of vulvovaginal candidiasis in pregnancy at three health centers in Burkina Faso. J Mycol Med. 2018; 28(1):186-92.

30. Araj GF, Asmar RG, Avedissian AZ. Candida profiles and antifungal resistance evolution over a decade in Lebanon. J Infect Dev Countries. 2015:9(9):997-1003

31. Guzel AB, Aydin M, Meral M, Kalkanci A, Ilkit M. Clinical characteristics of Turkish women with Candida krusei vaginitis and antifungal susceptibility of the C. krusei isolates. Infect Dis Obstet Gynecol. 2013;2013:698736.

32. Pappas PG, Kauffman CA, Andes DR, Clancy CJ, Marr KA, Ostrosky-Zeichner L, Reboli AC, Schuster MG, Vazquez JA, Walsh TJ, et al. Clinical practice guideline for the Management of Candidiasis: 2016 update by the Infectious Diseases Society of America. Clin Infect Dis. 2016;62(4):e1-50.

33. Eksi F, Gayyurhan ED, Balci I. In vitro susceptibility of Candida species to four antifungal agents assessed by the reference broth microdilution method. TheScientificWorldJournal. 2013;2013:236903.

34. Badiee P, Badali H, Diba K, Ghadimi Moghadam A, Hosseininasab A, Jafarian H, Mohammadi R, Mirhendi H, Najafzadeh MJ, Shamsizadeh A, et al. Susceptibility pattern of Candida albicans isolated from Iranian patients to antifungal agents. Curr Med Mycol. 2016;2(1):24-9.

35. Mokaddas EM, Al-Sweih NA, Khan ZU. Species distribution and antifungal susceptibility of Candida bloodstream isolates in Kuwait: a 10-year study. J Med Microbiol. 2007:56(Pt 2):255-9.

36. Whaley SG, Berkow EL, Rybak JM, Nishimoto AT, Barker KS, Rogers PD. Azole antifungal resistance in Candida albicans and emerging non-albicans Candida species. Front Microbiol. 2016;7:2173. 
37. Mendling W, Brasch J, Cornely OA, Effendy I, Friese K, Ginter-Hanselmayer G, Hof H, Mayser P, Mylonas I, Ruhnke M, et al. Guideline: vulvovaginal candidosis (AWMF 015/072), S2k (excluding chronic mucocutaneous candidosis). Mycoses. 2015;58(Suppl 1):1-15.

38. Rathod S, Vijayalakshmi S. Prevalence of vaginitis during pregnancy and its fetomaternal outcome in the rural setup. Int J Reprod Contracept Obstet Gynecol. 2016;5(6):1823-6.

39. Rasti S, Asadi MA, Taghriri A, Behrashi M, Mousavie G. Vaginal candidiasis complications on pregnant women. Jundishapur J Microbiol. 2014;7(2): e10078.

40. Zhang X, Liao Q, Wang F, Li D. Association of gestational diabetes mellitus and abnormal vaginal flora with adverse pregnancy outcomes. Medicine. 2018;97(34):e11891.

\section{Publisher's Note}

Springer Nature remains neutral with regard to jurisdictional claims in published maps and institutional affiliations.

Ready to submit your research? Choose BMC and benefit from:

- fast, convenient online submission

- thorough peer review by experienced researchers in your field

- rapid publication on acceptance

- support for research data, including large and complex data types

- gold Open Access which fosters wider collaboration and increased citations

- maximum visibility for your research: over $100 \mathrm{M}$ website views per year

At BMC, research is always in progress.

Learn more biomedcentral.com/submissions 